\title{
Nanocomposite solar cells - influence of particle concentration, size and shape on the device performance
}

\author{
C. Y. Kwong, ${ }^{1}$ A.B. Djurišić, ${ }^{2}$ P.C. Chui, ${ }^{1}$ W.K. Chan ${ }^{3}$
}

\author{
${ }^{I}$ Department of Electrical \& Electronic Engineering, University of Hong Kong, \\ Pokfulam Road, Hong Kong \\ ${ }^{2}$ Department of Physics, University of Hong Kong, Pokfulam Road, Hong Kong \\ ${ }^{3}$ Department of Chemistry, University of Hong Kong, Pokfulam Road, Hong Kong
}

\begin{abstract}
Solar cells based on poly(3-hexylthiophene) ( $\mathrm{P} 3 \mathrm{HT}): \mathrm{TiO}_{2}$ nanocomposite films were investigated. We studied the influence of the nanoparticle concentrations and different nanostructures (spherical particles with size $\sim 5 \mathrm{~nm}$ and $\sim 20$ $40 \mathrm{~nm}$, and rods with diameter $\sim 10 \mathrm{~nm}$ and length $\sim 40 \mathrm{~nm}$ ) on the performance of the nanocomposite solar cells. PL quenching and improved external quantum efficiency (EQE) was observed for all the nanocomposite devices compared to that of pristine P3HT solar cells. However, $\mathrm{TiO}_{2}(\sim 5 \mathrm{~nm}$ spheres $)$ and $\mathrm{TiO}_{2}$ rods showed only small improvement in EQE. The small improvement for the $5 \mathrm{~nm} \mathrm{TiO}{ }_{2}$ spheres was attributed to the lack of connectivity of nanoparticles for electron conduction. Therefore, the charge collection efficiency was limited. For $\mathrm{TiO}_{2}$ rods, the tendency of the rods to lie in the plane of substrates also limited the charge conduction and collection in the direction perpendicular to the substrates. Therefore, the improvement of the devices made by these nanoparticles was limited. For $\mathrm{TiO}_{2}(20-40 \mathrm{~nm}$ spheres) with optimal concentration, external quantum efficiency up to $15 \%$ and AM1 power conversion efficiency of $0.42 \%$ were obtained. The improvement in the efficiency was related to the improved morphology of the film and was attributed to the formation of percolation paths of $\mathrm{TiO}_{2}$ for electron conduction.
\end{abstract}

Keywords: organic solar cells, nanocomposite

\section{INTRODUCTION}

Polymer photovoltaic has been one of the main research topics in the field of optoelectronics due to the simple and economic fabrication process. Up to $2.5 \%$ AM1.5 power conversion efficiency with bilayer polymer solar cells ${ }^{1}$ and 2.9\% AM1.5 power conversion efficiency with bulk-heterojunction structure ${ }^{2}$ have been reported. Although the power conversion efficiency has been significantly improved, it is still not high enough for any applications. The main reasons for the low power conversion efficiency are incomplete coverage of solar light spectrum, short exciton diffusion length and low carrier mobility. The coverage of solar light spectrum of the overall devices can be improved by using two organic materials with complementary absorption spectrum. Short exciton diffusion length limits the exciton dissociation efficiency since each photoexcited exciton has to diffuse to the organic-organic interface or organic-metal interface for dissociation into free carriers which are subsequently collected by the appropriate electrodes for photocurrent generation. This limitation can be overcome by using bulk-heterojunction structure. In bulk-heterojunction solar cells, the active layer is made by two different materials, such as $\mathrm{CuPc}\left(\right.$ copper phthalocyanine): $\mathrm{C}_{60}($ fullerene) mixed layer in molecular solar cells ${ }^{3-5}$ and polymer:PCBM(methanofullerene) composite layer in polymer solar cells. ${ }^{6,7}$ The two materials inside the active layer form large interface area for exciton dissociation. Moreover, by proper adjusting the ratio between the two materials, the carrier collection efficiency can be increased because of the formation of percolation path of each material. ${ }^{5,8}$ These factors will help to increase the power conversion efficiency. However, low mobility, especially the electron mobility (with the exception of $\mathrm{C}_{60}$ and its derivatives), is the intrinsic property of organic materials. In most organic materials, the hole mobility is much higher ( 2 order of magnitudes) than the electron mobility. Since the photocurrent consists of hole-current and electron-current, low electron mobility will limit the magnitude of photocurrent. The use of inorganic nanoparticles with high electron mobility along with hole-conducting polymer in bulk-heterojunction solar cells seems to be promising to overcome this intrinsic limitation of organic materials while keeping the ease of solution fabrication process. 
Huynh et al. have demonstrated 1.7\% AM1.5 power conversion efficiency by using poly(3-hexylthiophene) (P3HT):CdSe nanorods composite as the active layer of their devices. ${ }^{9}$ They also found that the shape and size of the nanoparticles affected the device performance. The highest external quantum efficiency (EQE) of $55 \%$ at $485 \mathrm{~nm}$ was obtained with $7 \mathrm{~nm}$ x $60 \mathrm{~nm}$ nanorods. ${ }^{9}$ However, majority of the solar cells using nanocomposite active layer reported very low efficiency. ${ }^{10-12}$ The use of carbon nanotubes (CNT) in bulk-heterojunction solar cells seems promising to improve electron conduction in the active layer. However the power conversion efficiency was only $0.06 \%$. ${ }^{11}$ The low efficiency of their devices may due to the tendency of CNT to lie in the plane of substrate. This will limit the electron conduction and result in low charge collection efficiency. Breeze et al. have investigated comprehensively the influence of $\mathrm{TiO}_{2}$ morphology to the devices performance. ${ }^{13}$ They found that the devices with nanocomposite active layer showed only $6.4 \% \mathrm{EQE}$ and $0.002 \%$ power conversion efficiency. The main reasons for the low power conversion efficiency might be the discontinuity of $\mathrm{TiO}_{2}$ nanoparticles. The isolated nanoparticles within the polymer films trapped the electrons which subsequently recombined with the holes nearby in the polymer. Recently, Sun et al. ${ }^{14}$ showed $1.8 \%$ AM1.5 power conversion efficiency by using CdSe nanoparticles. The CdSe tetrapods could prevent the lying of nanoparticles in the plane of polymer films. This enhanced the electron conduction in the direction perpendicular to the plane of the films and enhanced electron collection efficiency.

In this work, we have investigated the influence of nanoparticle concentrations and different nanostructures on the performance of polymer nanoparticle bulk-heterjunction solar cells. The solar cell structure is ITO/PEDOT:PSS(poly(ethylenedioxythiophene) blended with poly(styrenesulfonate))/P3 $\mathrm{HT}: \mathrm{TiO}_{2} / \mathrm{Al}$. Photoluminescence (PL) and atomic force microscopy (AFM) are used to characterize the optical properties and film morphology respectively. The solar cells performance is characterized by current-voltage (I-V) measurement in the dark and under $100 \mathrm{~mW} / \mathrm{cm}^{2}$ AM1 illumination and by measuring the EQE at different wavelength. The paper is organized as follows. In the following section, experimental details are given. In Section 3, obtained results are presented and discussed. Finally, conclusions are drawn.

\section{EXPERIMENTAL DETAILS}

Indium tin oxide (ITO) coated glass substrates with sheet resisteance of $10 \mathrm{ohms} / \mathrm{square}$ were supplied by China South Glass Holding Co. Ltd., Shenzhen, China. Patterned ITO glass substrates were cleaned in ultrasonic bath using toluene, acetone, ethanol, and de-ionized water. PEDOT:PSS (Elec grade) and regioregular P3HT were purchased from Aldrich. $\mathrm{TiO}_{2}$ nanoparticles with particle size 20-40 nm (solid spherical particles, anatase/rutile structures), denoted as $\mathrm{TiO}_{2}$-large spheres, were purchased from Nanopowder Enterprises. Rutile- $\mathrm{TiO}_{2}$ nanorods with $40 \mathrm{~nm}$ in length and 10 $\mathrm{nm}$ in diameter (denoted as $\mathrm{TiO}_{2}$-rods) and anatase $\mathrm{TiO}_{2}$ spheres with size of $5 \mathrm{~nm}$ (denoted as $\mathrm{TiO}_{2}$-small spheres) were purchased from Nanostructured and Amorphous Materials Inc. All materials were used as received. $30 \mathrm{~nm}$ of PEDOT:PSS was spin-coated on top of the ITO substrates. The films were then baked at $110^{\circ} \mathrm{C}$ under vacuum for $24 \mathrm{~h}$. After that, $\sim 100 \mathrm{~nm}$ of $\mathrm{P} 3 \mathrm{HT}$ layer or $\mathrm{P} 3 \mathrm{HT} / \mathrm{TiO}_{2}$ nanocomposite layer were spin-coated on top of the PEDOT:PSS coated ITO substrates. The P3HT solution were prepared with xylene and kept at $50^{\circ} \mathrm{C}$ in order to obtain a concentration high enough $(\sim 10 \mathrm{mg}$ of $\mathrm{P} 3 \mathrm{HT} / \mathrm{ml})$ for spin-coating $100 \mathrm{~nm}$ thick films. Then different amount (by weight $\%$ ) of $\mathrm{TiO}_{2}$ were added to the solution and kept in a warm ultrasonic bath for dispersion before fabrication. All $\mathrm{P} 3 \mathrm{HT}$ or $\mathrm{P}_{3} \mathrm{HT}$ :TiO films were baked at $110^{\circ} \mathrm{C}$ under vacuum for another $24 \mathrm{~h}$. Finally, Al electrode was deposited thermally under high vacuum $\left(10^{-6}\right.$ torr). The thickness of all the layers has been verified by step profiler. The structure of the films for AFM measurement was ITO/PEDOT:PSS/P3HT:TiO 2 which was the same structure as the solar cells..

The room temperature photoluminescence of the $\mathrm{P} 3 \mathrm{HT}: \mathrm{TiO}_{2}$ composite films and the pure P3HT film was measured using $\mathrm{HeCd}$ laser excitation source $(325 \mathrm{~nm})$. AFM of the films was measured by Digital Instruments Nanoscope III operating in dynamic mode. Current-voltage (I-V) characteristic was measured by computer driven Keithley 2400 source-measurement unit. For white light efficiency measurements, Oriel 66002 solar simulator with AM1 filter was used. For EQE measurement, Oriel Cornerstone monochromator was used.

\section{RESULTS AND DISCUSSION}

In our previous studies, we found that the device performance of ITO/PEDOT:PSS/P3HT:TiO2(20-40 nm)/Al was very sensitive to the solvents used. The obtained power conversion efficiency ranged from $0.42 \%$ (spin-coated from 
xylene) to $0.033 \%$ (spin-coated from tetrahydrofuran). ${ }^{15}$ This can be attributed to preferential solvation of $\pi$-electron conjugated segments in aromatic solvents. ${ }^{16}$ Therefore, the devices and the composite films for PL and AFM measurements in this work are spin-coated from xylene solutions.

Figure 1 shows the energy diagram and the device structure of the solar cells. The energy levels of the materials were published in Refs. ${ }^{17-19}$ As shown in Figure 1, it is energetically favorable for photoexcited electrons in P3HT to transfer to $\mathrm{TiO}_{2}$ nanoparticles. Figure 2 shows the PL of P3HT:TiO 2 film with different nanostructures at optimal concentration as well as the pristine P3HT film. The PL intensity of all P3HT: $\mathrm{TiO}_{2}$ nanocomposite films is quenched compared to that of pristine P3HT films. This further supports the hypothesis that photoexcited electrons are transferred to the $\mathrm{TiO}_{2}$ nanoparticles. This quenching of PL intensity also indicates an improvement in exciton dissociation efficiency due to large interface area formed between the nanoparticles and P3HT. The degree of PL quenching of both spherical nanoparticles is significant while that of nanorods is mild. This shows that exciton dissociation should be more efficient in composite films containing spherical nanoparticles than films containing nanorods. It can be explained as larger interfacial area formed between spherical nanoparticles and P3HT than that formed between nanorods and P3HT. The improvement in exciton dissociation for all nanocomposite films is expected to have beneficial effects to the performance of solar cells.

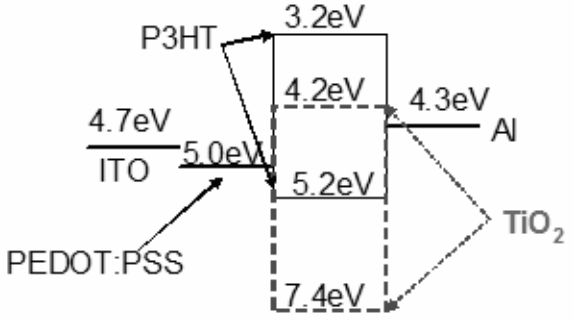

a) Energy diagram of nanocomposite solar cells

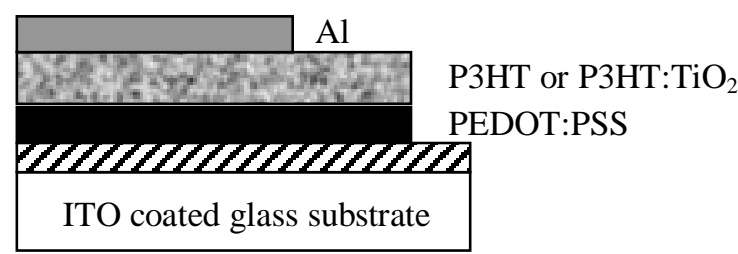

b) Solar cell structure

Figure 1

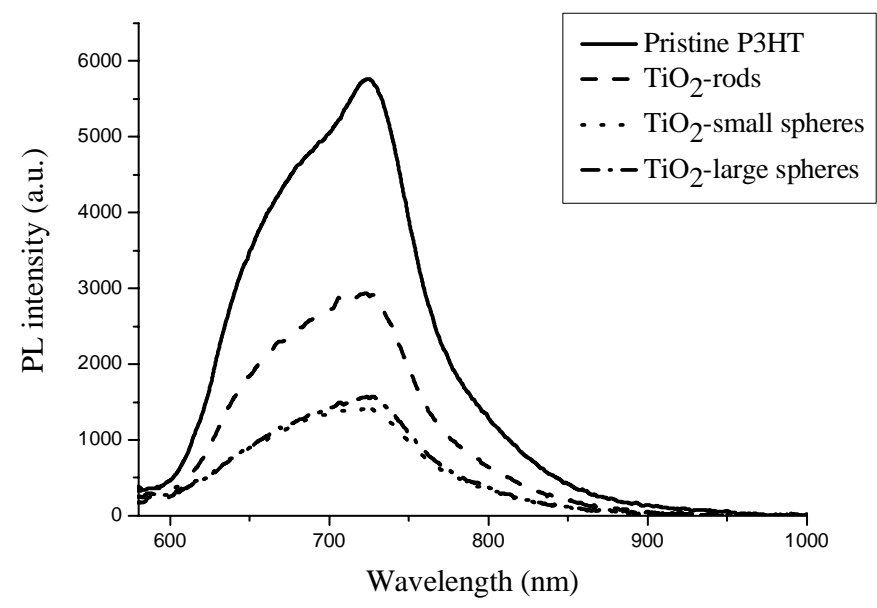

Figure 2. PL intensity of P3HT: $\mathrm{TiO}_{2}$ with different nanostructure at optimal concentration. 
We have investigated the influence of the concentration of different $\mathrm{TiO}_{2}$ nanoparticles on the solar cell performance. As expected, the best performance of devices fabricated with different $\mathrm{TiO}_{2}$ nanostructure is obtained at different optimal concentration. The concentration to achieve the best device performance is $40 \%$ for $\mathrm{TiO}_{2}$-rods, while it is $60 \%$ for both $\mathrm{TiO}_{2}$-large spheres and $\mathrm{TiO}_{2}$-small spheres. Similar trends with concentration have been observed for different nanoparticles (Figure 3). The most sensitive parameter is short circuit current density $\left(\mathrm{I}_{\mathrm{sc}}\right.$ ). Figure 3 shows the normalized short circuit current density of devices made with different $\mathrm{TiO}_{2}$ at different concentrations. At low concentrations, the short circuit current density is not much improved or even worse compared to that of the pristine P3HT devices. This is attributed to the lack of connectivity of nanoparticles within the polymer matrix. Therefore, the "dead end" of $\mathrm{TiO}_{2}$ paths may trap the electrons which may recombine with the holes in the polymer matrix. ${ }^{20}$ Thus, the performance of the devices is limited by charge collection efficiency even though exciton dissociation efficiency has been improved. $\mathrm{I}_{\mathrm{sc}}$ keeps increasing until it has reached a maximum value at optimal concentration of $\mathrm{TiO}_{2}$. At this point, $\mathrm{TiO}_{2}$ nanoparticles form percolation path for electron conduction to the cathode while holes are transported to the anode through the polymer matrix. At this concentration, the power conversion efficiency of the devices is significantly improved. For the best device, the devices fabricated with $\mathrm{P} 3 \mathrm{HT}: \mathrm{TiO}_{2}$-large spheres $(60 \%$ by weight $)$, we obtain $2.76 \mathrm{~mA} / \mathrm{cm}^{2}$ for short circuit current density, $0.44 \mathrm{~V}$ for open circuit voltage and 0.36 for fill factor. This results in $0.42 \%$ AM1 power conversion efficiency at $100 \mathrm{~mW} / \mathrm{cm}^{2}$ light intensity compared to the value of $0.059 \%$ with pristine P3HT devices. However, with further increase of the concentration of $\mathrm{TiO}_{2}$ nanoparticles, the film becomes too rough and nonuniform resulting in significant decrease of $\mathrm{I}_{\mathrm{sc}}$. Therefore, devices with good performance cannot be fabricated. At too low or too high concentration, the nanocomposite device performance is only comparable to or even worse (in some cases) than the performance of the devices with pristine P3HT.

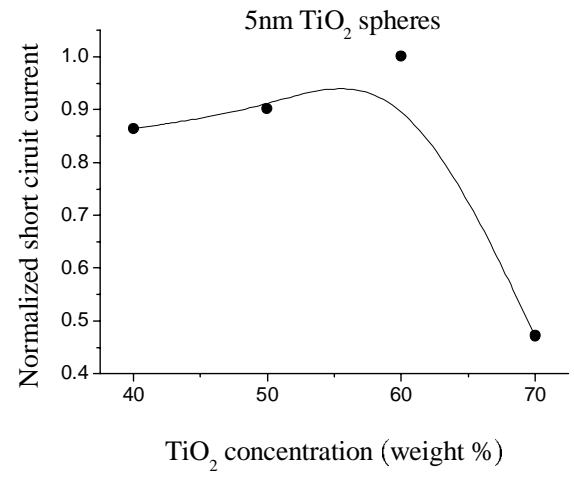

a)

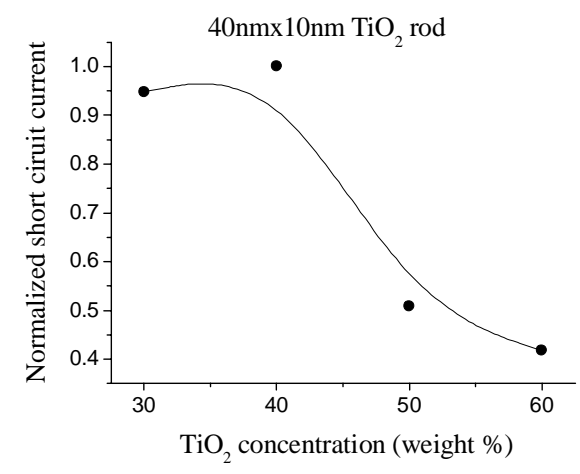

c)

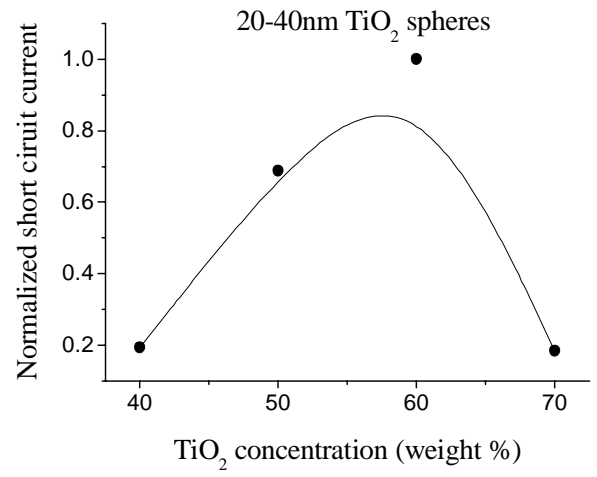

b)

Figure 3. Normalized short circuit current density of different nanostructures

a) $5 \mathrm{~nm}$ anatase $\mathrm{TiO}_{2}$ spheres

b) $20-40 \mathrm{~nm}$ anatase and rutile $\mathrm{TiO}_{2}$ spheres

c) $10 \mathrm{~nm}$ by $40 \mathrm{~nm}$ rutile $\mathrm{TiO}_{2}$ nanorods 
Figure 4 shows EQE of the devices with different $\mathrm{TiO}_{2}$ nanostructures at optimal concentration. The EQE of $\mathrm{TiO}_{2}$-rods devices, $\mathrm{TiO}_{2}$-small spheres devices and pristine P3HT devices has been multiplied by a factor of 10 . The EQE of all devices with nanoparticles is higher than that of the devices with pristine P3HT. It is because the exciton dissociation efficiency is improved in the nanocomposite active layer by the large interface area formed between the nanoparticles and P3HT. However, exciton dissociation efficiency is not the only limiting factor in organic solar cells. The performance of $\mathrm{TiO}_{2}$-rods devices is better than that of $\mathrm{TiO}_{2}$-small spheres devices even though the degree of PL quenching of $\mathrm{TiO}_{2}$-rods films is smaller than that of $\mathrm{TiO}_{2}$-small spheres films. The reason is that it is easier to form nanoparticle networks for electron conduction using nanorods with $40 \mathrm{~nm}$ in length than using spherical nanoparticles with $5 \mathrm{~nm}$ size. Therefore, the higher EQE obtained can be attributed to the higher charge collection efficiency of $\mathrm{TiO}_{2}$ rods devices than that of $\mathrm{TiO}_{2}$-small spheres devices. However, the performance is still not improved significantly. The reason may be due to the tendency of the nanorods to lie parallel to the plane of the substrates as proposed by Sun et $a l .{ }^{14}$. This limits the electron conductivity in the direction perpendicular to the substrate. Therefore, the charge collection is still limited. To overcome this problem, we have also fabricated devices with spherical $\mathrm{TiO}_{2}$ with size of 20-40 nm, similar to the length of nanorods. The EQE of $\mathrm{P} 3 \mathrm{HT}: \mathrm{TiO}_{2}$-large spheres devices is order of magnitude higher that of $\mathrm{P} 3 \mathrm{HT}: \mathrm{TiO}_{2}$-small spheres cells and $\mathrm{P} 3 \mathrm{HT}: \mathrm{TiO}_{2}$-rods cells. The better performance can be attributed to higher electron collection efficiency. As for spherical nanoparticles, there is no preferential alignment of nanoparticles to the substrate as it is the case for nanorods. Therefore, the electron conduction in the direction perpendicular to the plane of substrate has been improved. Therefore, electron collection efficiency is improved. This is in agreement with other report in which they found that devices made with CdSe tetrapods gave better electron collection efficiency compared to devices made with CdSe nanorods. ${ }^{14}$ Comparing devices made with different spherical nanoparticles, it is easier to form nanoparticle networks with larger spheres $(20-40 \mathrm{~nm})$ than with small spheres $(5 \mathrm{~nm})$ for electron conduction. Therefore, $\mathrm{TiO}_{2}$-large spheres devices shows much higher EQE than that of $\mathrm{TiO}_{2}$-small spheres devices even though both films made by spherical nanostructures have similar degree of PL quenching. For the $\mathrm{TiO}_{2}$-large spheres devices, we obtain EQE of $15 \%$ peaked at $490 \mathrm{~nm}$.

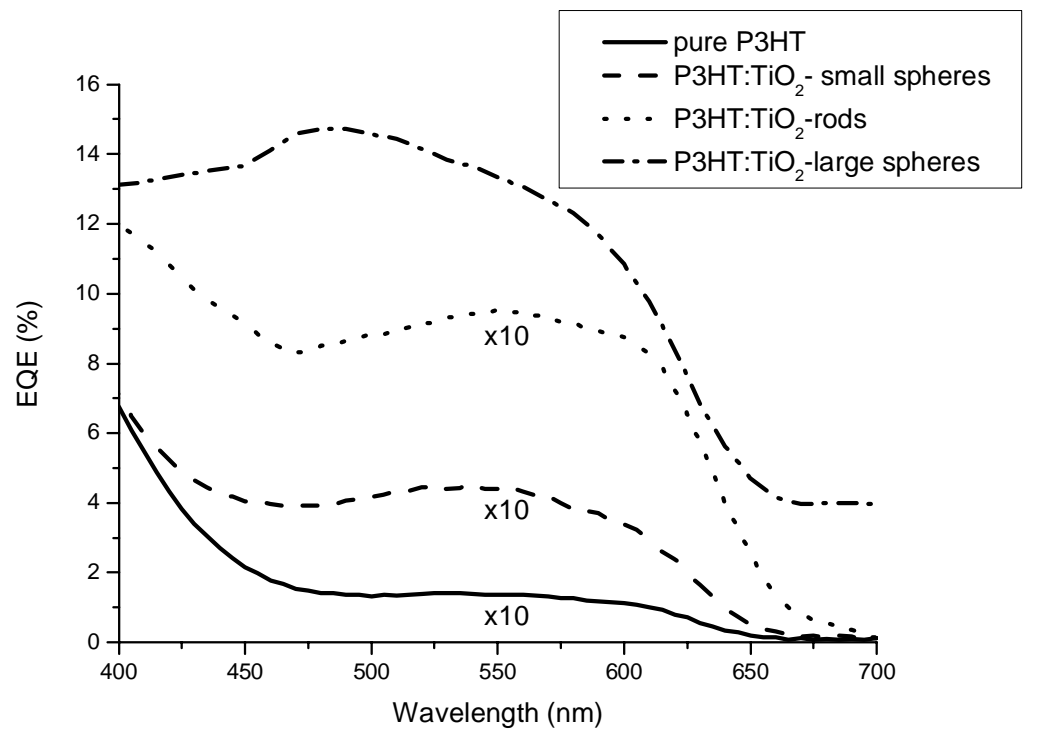

Figure 4. EQE of solar cells with different $\mathrm{TiO}_{2}$ nanoparticles, the signal of device with pristine P3HT, $\mathrm{P} 3 \mathrm{HT}$ :TiO $\mathrm{T}_{2}$-rod and P3HT: $\mathrm{TiO}_{2}$-small spheres has been multiplied by 10 .

It has also been shown that the film morphology and phase-separation of the two constituents affect the device performance seriously. ${ }^{21}$ Therefore, we have characterized the nanocomposite films using AFM. Figure 5 shows the topography (left) and phase contrast image (right). The topography of all composite films is similar. The rms. roughness 
of all composite films is around $\sim 20 \mathrm{~nm}$. However, large differences have been observed for their phase contrast images. For $5 \mathrm{~nm} \mathrm{TiO}_{2}$ spheres, the nanoparticles are distributed separately in the polymer matrix. Therefore, the electron conduction through the nanoparticles is limited due to the absence of connectivity in nanoparticle networks. This causes the devices to have poor performance. For $\mathrm{TiO}_{2}$-rods, the connectivity of $\mathrm{TiO}_{2}$ domains in the nanoparticle network is improved. This improves the electron conduction of the composite films. However, it is suspected that the nanorods improve electron conductivity only in the direction parallel to the plane of substrate due to the tendency of the nanorods to align parallel to the substrate. Therefore, the improvement of $\mathrm{TiO}_{2}$-rods devices is not very significant. For 20-40 nm $\mathrm{TiO}_{2}$ spheres, the nanoparticles form a larger domain for electron conduction. It is also expected that the improvement of electron conduction is in all direction for a spherical particles. Therefore, devices with these larger spherical nanoparticles show significant improvement.
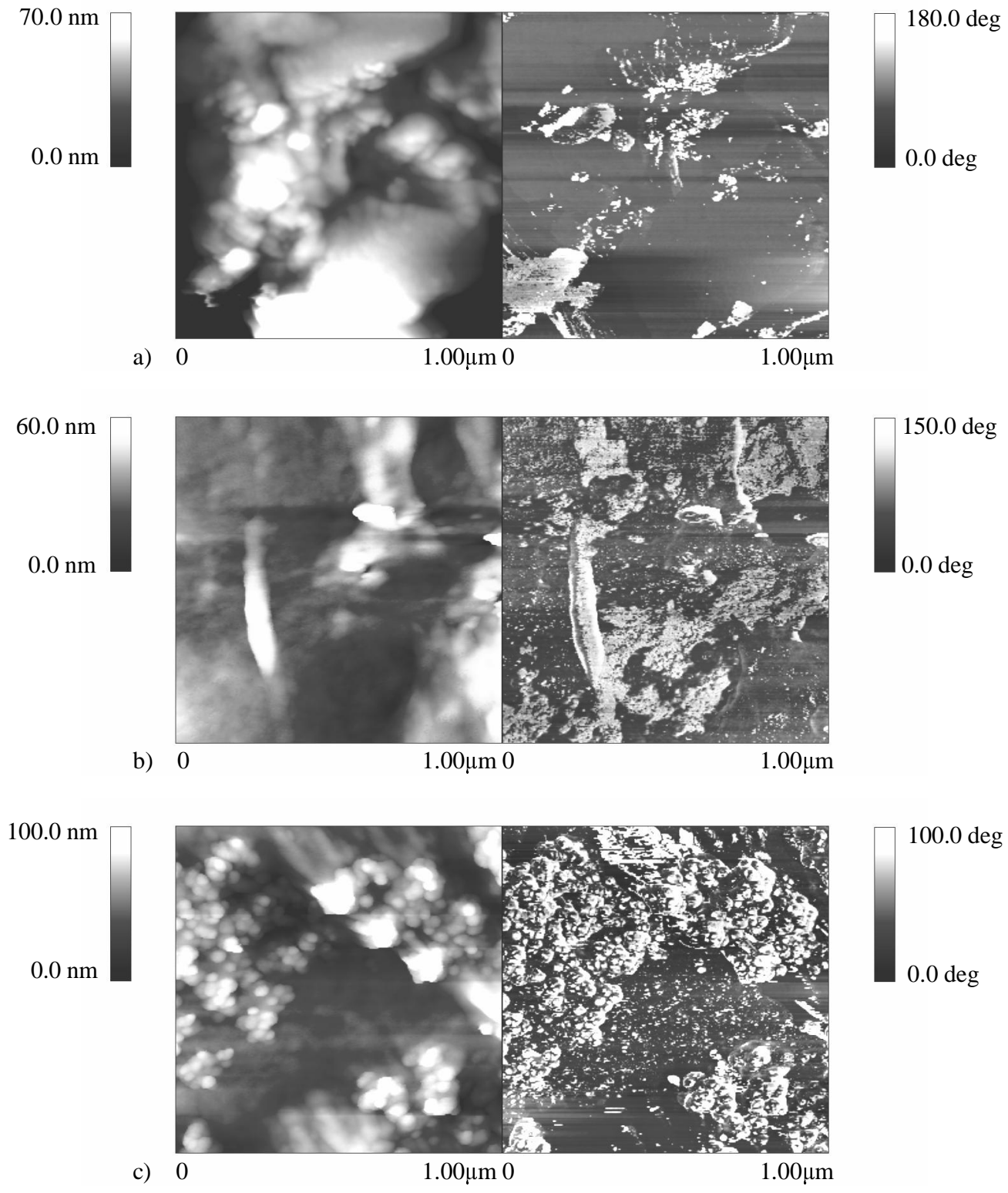

Figure 5. AFM topography (left) and phase contrast (right) images of $\mathrm{P} 3 \mathrm{HT}: \mathrm{TiO}_{2}$ films on top of PEDOT:PSS covered ITO substrates. a) $\mathrm{TiO}_{2}$-small spheres, b) $\mathrm{TiO}_{2}$-rods and c) $\mathrm{TiO}_{2}$-large spheres 


\section{CONCLUSIONS}

We have investigated the influence of size and shape of different $\mathrm{TiO}_{2}$ nanoparticles and their concentration to the performance of organic solar cells. PL quenching is observed for all nanocomposite films which indicates higher exciton dissociation efficiency. Therefore, we have also found that the performance of the devices with nanocomposite layer is better than that of the devices made by pristine P3HT. However, only small improvement is obtained with $\mathrm{TiO}_{2}-$ rods devices and $\mathrm{TiO}_{2}$-small spheres devices. This is due to limited electron conduction in the direction perpendicular to the substrates. This will in turn limit the electron collection efficiency and the power conversion efficiency. The best performance is obtained with $20-40 \mathrm{~nm} \mathrm{TiO}$ spheres consisting of anatase and rutile structure at $60 \%$ concentration by weight. It is because the electron collection efficiency has been greatly improved due to the formation of continuous nanoparticle networks for electron conduction. The obtained AM1 power conversion efficiency and EQE are $0.42 \%$ and $15 \%$ (peaked at $490 \mathrm{~nm}$ ), respectively. This performance is an order of magnitude larger than that of devices made with pristine P3HT.

\section{ACKNOWLEDGMENTS}

This work is partly supported by Research Grants Council of the Hong Kong Special Administrative Region, China (Project Nos. HKU7075/01P, 7056/02E, 7095/01P, 7096/02P and 7009/03P). The authors would like to thank Prof. G. K. Y. Chan and Ms. Crystal Cheung from the Department of Chemistry, the University of Hong Kong for the AFM measurements.

\section{REFERENCES}

1. S. E. Shaheen, C. J. Brabec, N. S. Sariciftci, F. Padinger, T. Fromherz and J. C. Hummelen, " $2.5 \%$ efficiency organic plastic solar cells", Appl. Phys. Lett., 78, pp. 841 - 843, Feb. 2001.

2. S. Alem, R. de Bettignies, J.M. Nunzi and M. Cariou, "Efficient polymer-based interpenetrated network photovoltaic cells", Appl. Phys. Lett., 84, pp. 2178 - 2180, Mar. 2004.

3. C. Y. Kwong, A. B. Djurišić, P. C. Chui and W. K. Chan, "CuPc/C 60 Solar Cells - Influence of the Indium Tin Oxide Substrate and Device Architecture on the Solar Cell Performance”, Jpn. J. Appl. Phys., 43, pp. 1305 - 1311, Apr. 2004.

4. S. Uchida, J. Xue, B. P. Rand and S. R. Forrest, "Organic small molecule solar cells with a homogeneously mixed copper phthalocyanine: $\mathrm{C}_{60}$ active layer", Appl. Phys. Lett., 84, pp. 4218 - 4220, May 2004.

5. S. Heutz, P. Sullivan, B. M. Sanderson, S. M. Schultes and T. S. Jones, "Influence of molecular architecture and intermixing on the photovoltaic, morphological and spectroscopic properties of CuPc-C 70 heterojunction", Solar Energy Materials and Solar Cells, 83, pp. 229 - 245, Jun. 2004.

6. S. E. Shaheen, R. Radspinner, N. Peyghambarian and G. E. Jabbour, "Fabrication of bulk heterojunction plastic solar cells by screen printing”, Appl. Phys. Lett., 79, pp. 2996 - 2998, Oct. 2001.

7. J. K. J. van Duren, X. Yang, J. Loos, C. W. T. Bulle-Lieuwma, A. B. Sieval, J. C. Hummelen and R. A. J. Janssen, "Relating the Morphology of Poly(p-phenylene vinylene)/Methanofullerene Blends to Solar-Cell Performance", Adv. Funct. Mater., 14, pp. 425 - 434, May 2004.

8. M. T. Rispens, A. Meetsma, R. Rittberger, C. J. Brabec, N. S. Sariciftci and J. C. Hummelen, "Influence of the solvent on the crystal structure of PCBM and the efficiency of MDMO-PPV:PCBM "plastic" solar cells", Chem. Commun., pp. 2116 - 2118, Aug. 2003.

9. W. U. Huynh, J. J. Dittmer and A. P. Alivisatos, "Hybrid Nanorod-Polymer Solar Cells", Science, 295, pp. 2425 2427, 2002.

10. C. L. Huisman, A. Goosens, and J. Schoo nman, "Preparation of a nanostructured composite of titanium dioxide and polythiophene: a new route towards 3D heterojunction solar cells", Synthetic Metals, 138, pp. 237 - 241 , Jun. 2003.

11. E. Kymakis, I. Alexandrou, and G. A. J. Amaratunga, "High open-circuit voltage photovoltaic devices from carbon-nanotube-polymer composites”, J. Appl. Phys., 93, pp. 1764 - 1768, Feb. 2003.

12. E. Arici, N. S. Sariciftci, and D. Meissner, "Hybrid Solar Cells Based on Nanoparticles of CuInS2 in Organic Matrices”, Adv. Funct. Mater., 13, pp. 165 - 171, Feb. 2003. 
13. A. J. Breeze, Z. Schlesinger, S. A. Carter and P. J.Brock, "Charge transport in TiO $\mathrm{T}_{2} \mathrm{MEH}-\mathrm{PPV}$ polymer photovoltaics", Phys. Rev. B, 64, pp. 125205-1 - 125205-9, Sep.2001.

14. B. Sun, E. Marx and N. C. Greeham, "Photovoltaic Devices Using Blends of Branched CdSe nanoparticles and Conjugated Polymers", Nano Lett., 3, pp. 961 - 963, Jun. 2003.

15. C. Y. Kwong, A. B. Djurišić, P. C. Chui, K. W. Cheng and W. K. Chan, "Influence of solvent on film morphology and device performance of poly(3-hexylthiophene): $\mathrm{TiO}_{2}$ nanocomposite solar cells", Chem. Phys. Lett., 384, pp. 372 - 375, Jan. 2004.

16. J. Liu, Y. Shi, and Y. Yang, "Solvation-Induced Morphology Effects on the Performance of Polymer-Based Photovoltaic Devices" Adv. Funct. Mater., 11, pp. 420 - 424, Dec. 2001.

17. M. K. Fung, S. L. Lai, S. W. Tong, M. Y. Chan, C. S. Lee, S. T. Lee, W. W. Wu, M. Inbasekaran, and J. J. O'brien, "Anode modification of polyfluorene-based polymer light-emitting devices", Appl. Phys. Lett., 81, pp. 1497 - 1499, Aug. 2002.

18. A. C. Arango, S. A. Carter, and P. J. Brock, "Charge transfer in photovoltaics consisting of interpenetrating networks of conjugated polymer and $\mathrm{TiO}_{2}$ nanoparticles" Appl. Phys. Lett., 74, pp. 1698 - 1670, Mar. 1999.

19. R. Valaski, L. M. Moreira, L. Micaroni, and I. A. Hümmelgen, "Charge injection and transport in electrochemical films of poly(3-hexylthiophene)", J. Appl. Phys., 92, pp. 2035 - 2040, Aug. 2002.

20. J. S. Salafsky, "Exciton dissociation, charge transport, and recombination in ultrathin, conjugated polymer-TiO nanocrystal intermixed composites", Phys. Rev. B, 59, pp. 10885 - 10894, Apr. 1999.

21. W.U. Huynh, J.J. Dittmer, W.C. Libby, G.L. Whiting, A.P. Alivisatos, "Controlling the Morphology of Nanocrystal-Polymer Composites for Solar Cells", Adv. Funct. Mater., 13, pp. 73 - 79 , Jan. 2003. 